\title{
PASSO A PASSO NA IMPLANTAÇÃO DO PROTOCOLO DE HIGIENE DE MÃOS
}

\section{STEP BY STEP IN THE IMPLANTATION THE PROTOCOL'S HANDS HYGIENE}

Recebido em: 15/04/2018.

Aceito em: 13/09/2018.
Rafaella de Menezes Leuthier Vanessa Maria Jurema Furtado Carvalho² Dostoievsky Ernesto de Melo Andrade ${ }^{3}$ Karla Fernandes de Albuquerque ${ }^{4}$ Tatiana Pimentel de Andrade Batista ${ }^{5}$

Maria Alenita de Oliveira ${ }^{6}$

\section{RESUMO}

A implantação do Núcleo de Segurança do Paciente visa melhorar a assistência do paciente através do aprimoramento da gestão de risco, incorporação de protocolos institucionais e promoção da cultura da segurança do paciente. Dentre estes protocolos, destaca-se o de Higiene de Mãos (HM). Objetivo: descrever as etapas do processo de implantação de um Protocolo de Higiene em um Hospital filantrópico no município de João Pessoa. Método: trata-se de um estudo descritivo e de abordagem quantitativa, realizado em um hospital filantrópico da cidade de João Pessoa, Paraíba. Resultados: foi elaborado um guia com base no material disponível na - Agência de Vigilância Sanitária (ANVISA) e na Organização Mundial da Saúde (OMS) para a implantação do protocolo de higiene de mãos considerando o planejamento através da ferramenta de gestão $5 \mathrm{~W} 2 \mathrm{H}$. A avaliação do protocolo de higiene foi orientada a partir do guia da OMS que inclui a infraestrutura como existências de pias, a educação sobre o tema,

$\overline{1 \text { Graduanda }}$ em Medicina pelo Centro Universitário de João Pessoa (UNIPÊ). E-mail: rafaella.leuthier@gmail.com

2 Graduanda em Medicina pelo Centro Universitário de João Pessoa (UNIPÊ). E-mail: vanessa_cb@hotmail.com

3 Mestre em Direito pelo Centro Universitário de João Pessoa (UNIPÊ). Docente do Centro Universitário de João Pessoa (UNIPÊ) e da Faculdade Maurício de Nassau. E-mail: dosto11@hotmail.com

4 Doutora em Ciências da Saúde pela Universidade Federal do Rio Grande do Norte (UFRN). Docente do Centro Universitário de João Pessoa (UNIPÊ). E-mail: karlaalbuq@ hotmail.com

5 Graduanda em Medicina pelo Centro Universitário de João Pessoa (UNIPÊ). E-mail: tatianabatista.infectologia@gmail.com

6 Doutora em Medicina pela Universidade Federal de São Paulo (UNIFESP). Docente do Centro Universitário de João Pessoa (UNIPÊ). E-mail: alenitaoliveira@uol.com.br 
cartazes informativos e clima institucional. Através desta ferramenta, o hospital onde ocorreu a experiência teve seu nível de higiene de mãos considerado inadequado. Após análise das condições do ambiente foi elaborado um plano de ação para a implantação do protocolo de higiene de mãos e confecção dos indicadores para a sua monitorização. Conclusão: o conhecimento de ferramentas de gestão e instrumentos que favoreçam a sistematização das ações necessárias leva a agilidade na melhoria do processo, sendo resultado do esforço compartilhado de toda a equipe, implicando na mudança de postura comportamental do grupo.

Palavras-chave: Higiene das mãos. Segurança do paciente. Infecção hospitalar. Conhecimentos, atitudes e prática em Saúde.

\section{ABSTRACT}

Introduction: The establishment of the Patient Safety Programs is an attempt to improve patient safety by managing risk, implementation of safety protocols and emphasis on creating a culture of patient safety. Objective: the diffusing of information for the facilitation and integration of the steps necessary for the embedding of a protocol for hands hygiene. Method: a descriptive study combined with a quantitative approach carried out at a charitable hospital in the city of João Pessoa, Paraiba. Results: A guide for the implementation of a protocol for hands hygiene was produced based on the material available in Agencia de Vigilancia Sanitaria (ANVISA and in Organização Mundial da Saude (OMS) incorporating $5 \mathrm{~W} 2 \mathrm{H}$ as a planning tool and using as a OMS'guide for evaluation of the proposed structure with reference to such infrastructure as availability of sinks, education, information posters and institutional culture. After an analysis of the extant conditions, its level of hand hygiene was considered. An action plan was produced for the implementation of a protocol for hands hygiene and the establishment of indicators for a monitoring of the said. Conclusion: A knowledge of administrative tools and instruments that help systematize the necessary actions identified in the study are major positive factors in improving the process. A shared effort of the whole team was involved in effecting a behavioral change in the study group.

Keywords: Hygiene of hands. Patient safety. Hospital infection. Knowledge, attitudes and practice in Health.

\section{INTRODUÇÃO}

Em 25 de julho de 2013, a Agência Nacional de Vigilância Sanitária (ANVISA) adotou a resolução da diretoria colegiada (RDC) $n^{\circ} 36$, que instituiu a obrigatoriedade 
de implantação do Núcleo de Segurança do Paciente (NSP) em serviços de saúde. A implantação do NSP visa melhorar a assistência do paciente através do aprimoramento da gestão de risco, da incorporação de protocolos institucionais e da promoção da cultura da segurança do paciente (BRASIL, 2013).

O Plano de Segurança do Paciente em Serviços de Saúde (PSP) é uma ferramenta que norteia as ações e estratégias necessárias para executar ações em busca das melhorias normatizadas pela $\mathrm{RDC} \mathrm{n}^{\circ} 36$. Um dos grandes desafios para a implantação deste plano se encontra nas mudanças de processos de trabalho das equipes de saúde. Muitos profissionais, apresentam-se resistentes a aceitar os novos processos de trabalho tendo em vista seus modelos mentais prévios e já incorporados (FIGUEIREDO, 2011).

Neste cenário, gestores de saúde relatam dificuldade em instituir de fato e de direito as ações do NSP. Apontam algumas dificuldades como desconhecimento da gestão de processos de melhoria, falta de infraestrutura e insumos, falta de envolvimento, entrosamento e da integração das equipes.

Este trabalho tem como objetivo descrever as etapas de um processo de implantação de um Protocolo de Higiene em um Hospital filantrópico no município de João Pessoa - PB.

A higiene das mãos constitui a meta de número cinco preconizada pela ANVISA a ser incorporada uma vez que esta medida está intrinsecamente relacionada à redução das Infecções Relacionadas à Assistência à Saúde (IRAS), um dos desafios em saúde pública em nível mundial.

Publicações nacionais que mostrem medidas exitosas no campo da segurança do paciente, assim como a forma de implantá-las, servem de modelo para auxiliar a atuação de outros NSP, principalmente considerando os problemas enfrentados nos hospitais públicos brasileiros.

\section{REVISÃO BIBLIOGRÁFICA}

Historicamente as infecções hospitalares, iniciaram-se com a criação dos primeiros hospitais, em 325 d.C., durante o Concílio de Nicéia. Neste período as doenças não eram separadas por gravidade e nem havia técnicas de assepsia que evitassem a propagação de infecções. Apesar já existir associação entre os hospitais e a transmissão de infecções, foi apenas no século XIX que Ignaz Semmelweis evidenciou a importância da lavagem das mãos, ao relatar a diminuição na taxa de infecção puerperal, quando introduziu o uso de solução clorada entre os procedimentos de necropsias realização de partos (BRASIL, 2009).

Nesta mesma época, foi implementado por Oliver Wendell Holmes, a lavagem das mãos para o controle de infecções (PITTET et al, 2006; HASS; LARSON, 2007).

Em 1846, a enfermeira Florence Nightingale, modificou as bases da assistência de enfermagem através de ações no hospital de Scutari na Guerra da Criméia. O hospital que atendia feridos de guerra no qual adotou ações rigorosas de higiene pessoal e do ambiente 
assistencial como o isolamento de áreas limpas das sujas o que reduziu a taxa de mortalidade de $33 \%$ para $2 \%$ (BARALD, 2015).

Dessa forma, a informação sobre o impacto e benefícios da higienização das mãos na prevenção de infecções vem sendo construído historicamente e comprovado com o aperfeiçoamento dos métodos científicos, sobretudo, no século XX. Entretanto, o desafio da adesão a essa medida ainda perdura nos dias de hoje (PINTO, 2010). As IRAS constituem importante problema de saúde pública e desafiam os avanços alcançados na área da saúde. Os países desenvolvidos apresentam índices de 5 a $10 \%$ dos pacientes hospitalizados com IRAS, sendo que nos Estados Unidos (EUA) são registradas aproximadamente dois milhões de IRAS relacionadas a iatrogenias na assistência em saúde (HADDAD, 2016). Aproximadamente 30\% das infecções que se relacionam à assistência são preveníveis por medidas simples e de baixo custo, sendo a lavagem correta das mãos pelos profissionais de saúde a mais efetiva delas (SOUSA, 2016).

A importância da prática de higiene de mãos (HM) baseia-se na capacidade que as mãos têm de abrigar microrganismos e de transferir esses agentes de uma superfície para outra seja por contato direto (pele com pele) ou indireto (por meio de objetos) (PRIMO, 2010; BARALD, 2015).

As IRAS são adquiridas pelos pacientes durante a ocasião em que necessite ser submetido aos cuidados pelos profissionais de saúde (RIBEIRO,2017).

Estima-se que um em cada 20 pacientes internados nos hospitais desenvolvem durante sua internação alguma IRAS, o que as torna a complicação mais comum decorrente do cuidado. São responsáveis por elevadas taxas de morbidade e mortalidade, assim como o aumento do tempo de internamento e aumento da resistência dos microrganismos aos antimicrobianos. Isto acarreta aumento da fragilidade emocional dos pacientes e seus familiares, óbitos que poderiam ser preveníveis, além de terem grande impacto nos custos com a assistência à saúde (BELELA, 2017) (RIBEIRO,2017).

O Brasil ainda passa por uma realidade adversa do que se pode avaliar como satisfatório: deficiência de recursos humanos e materiais hospitalares nas instituições de saúde (especialmente nas públicas), ausência de Comissão de Controle de Infecção Hospitalar (CCIH) que sejam efetivamente atuantes na maioria dos hospitais, ou ainda, profissionais que exercem sua função sem conhecimento apropriado da atividade, resultando em altas taxas de infecção hospitalar, episódios de surtos difíceis de serem detectados em berçários e unidades de terapia intensiva, surgimento de bactérias resistentes a múltiplos antibióticos e elevado risco ocupacional (BATISTA, 2004)

A HM tem sido considerada como a medida mais simples e eficaz na prevenção das infecções. É uma intervenção diária, padronizada, de custo mínimo e com recomendações amparadas por fundamentação científica sólida. Entretanto, a adesão ao procedimento ainda é descrita mundialmente como insuficiente (MARRA, 2014). O controle dessas infecções através da lavagem cuidadosa e rotineira das mãos atende perfeitamente às requisições legais e éticas assim como mantem a segurança e a qualidade do serviço prestado ao cliente (PRIMO, 2010). 
A preocupação com a disseminação das infecções inquieta muitos pesquisadores, levando à realização de estudos que visem à monitoração da aderência dos profissionais de saúde às práticas de lavagem de mão, tendo como principal desafio propor estratégias que incentivem a adesão e a manutenção das taxas ideais desta recomendação (OLIVEIRA, 2011).

Ainda que estudos evidenciem a importância das mãos na transmissão das infecções relacionadas a assistência à saúde e os benefícios da sua higienização na diminuição das taxas de infecção, a adesão dos profissionais dos serviços de saúde a essa prática é baixa, geralmente, não ultrapassando 50\% (BATHKE,2013).

As justificativas se relacionam a problemas no ato da lavagem, como ressecamento da pele e lesões nas mãos causadas pelo sabão ou detergente. São comuns também queixas por outros motivos, como a perda de tempo e a interrupção de procedimentos de rotina e de cuidados com o paciente (BRASIL,1998; ANVISA, 2008; NEVES, 2009; PINTO, 2010; MARRA,2016).

A falta de cumprimento da HM, pelos profissionais de saúde, é um tema preocupante em todo o mundo. No Brasil, a ANVISA vem promovendo campanhas a fim de propor estratégias que busquem influenciar o incentivo a adesão à higienização das mãos entre os profissionais da saúde (ANVISA, 2012).

A observação direta das oportunidades de HM tem sido a abordagem mais utilizada e bem aceita pelos pesquisadores para avaliar o comportamento e a adesão dos profissionais de saúde às medidas de controle de infecção, sendo considerada pela OMS padrão ouro para monitoração dessa prática (OLIVEIRA, 2011).

\section{METODOLOGIA}

Este trabalho se trata de relato de experiência decorrida a partir das atividades desenvolvidas no Hospital Padre Zé, na cidade de João Pessoa, na Paraíba. Fundado em 1965, o Hospital Padre Zé, possui 60 leitos, de cunho filantrópico, sem fins lucrativos. É reconhecido pelo cuidado aos necessitados e, através do convênio com o Centro Universitário de João Pessoa (UNIPE), busca aliar o cuidado à expertise da academia. A busca pela qualificação do cuidado, um dos objetivos de ambas as instituições, foi então posto como prioridade sendo a implantação do protocolo de higiene de mãos um dos objetivos a ser alcançado dentro do plano de segurança do paciente proposto.

O hospital possui 16 setores, e um corpo funcional de mais de 263 funcionários, incluindo voluntários. Foram entrevistados os profissionais das equipes atuantes na instituiçao como enfermeiros, técnicos, médicos, nutricionistas, fisioterapeutas, higienização e administração. As ações foram realizadas no período de junho a novembro de 2016

As atividades desenvolvidas estavam vinculadas a um projeto de pesquisa aprovado pelo comitê de Ética do Centro Universitário João Pessoa, com o número 2.457.414, de acordo com Resolução No 466/12 do CNS/MS. 


\section{RESULTADOS E DISCUSSÃO}

As atividades de planejamento para a implantação do projeto ocorreram em oito etapas e foi desenvolvido através da ferramenta $5 \mathrm{~W} 2 \mathrm{H}$ (CARPINETTI, 2012). A $5 \mathrm{~W} 2 \mathrm{H}$ é uma ferramenta administrativa que pode ser utilizada em qualquer empresa a fim de registrar de maneira organizada e planejada como serão efetuadas as ações. Seu nome designa uma sigla que contém todas as iniciais dos processos em inglês, sendo: 1 - What (o que); 2 - Who (quem); 3 - When (quando); 4 - Where (onde); 5 - Why (por que); 1 - How (como); 2 - HowMuch (quanto). Esta ferramenta funciona como um mapeamento das atividades a serem desenvolvidas: o que será feito, como e quem fará o quê, quando, onde, e os custos relacionados.

Tabela 1.Plano de ação de uma das ações para a elaboração do protocolo de Higiene de Mãos.

\begin{tabular}{ll}
\hline Etapas & Plano de ação \\
\hline O que será feito & Elaboração do protocolo de higiene de mãos \\
Por que será feito & Padronizar as ações do protocolo \\
Como & Baseado no protocolo do Ministério da saúde \\
Quem & Coordenador do núcleo e CCIH \\
Quando & Até mês de setembro \\
Onde & Na sala do NSP \\
Custo associado & Papel, tinta e impressora \\
\hline
\end{tabular}

As seguintes ações foram planejadas e encontram-se descritas abaixo:

\section{Avaliação diagnóstica da situação da promoção da higiene de mãos:}

Foi realizada uma avaliação da situação da promoção da higiene de mãos vigente, através do guia de auto avaliação para a higiene de mãos na instituição de saúde (World Health Organization, 2010).

O Guia de auto avaliação para a Higiene das Mãos está dividido em cinco componentes e 27 indicadores. Os cinco componentes refletem os cinco elementos da Estratégia Mundial da OMS para a Melhoria de Higiene das Mãos (ANVISA, 2008) e os 27 indicadores foram selecionados para representar os elementos chave de cada componente.

Os cinco componentes avaliados incluem os abaixo citados:

a) Mudança no sistema: que engloba aspectos relacionados a infraestrutura como existência de pias, dispensadores de álcool gel etc.

b) Formação e educação: como treinamento e material didático sobre o tema

c) Avaliação e retroalimentação: se há, por exemplo, auditorias, controle de consumo de sabonete e álcool gel

d) Cartazes informativos

e) Clima institucional: engajamento dos líderes e profissional qualificado para o treinamento 
Baseado na pontuação obtida para os cinco componentes, a instituição é classificada em um dos quatro níveis de prática e promoção da higiene das mãos, a saber: inadequado, básico, intermédio e avançado.

Com base nestes cinco componentes, a instituição do trabalho foi classificada em relação ao nível de higiene de mãos como inadequada. A partir deste resultado e considerando as deficiências observadas na instituição, foram desenvolvidas as seguintes medidas para promover as melhorias a serem implementadas no hospital

\section{Infraestrutura:}

Com base no diagnóstico de infraestrutura foi recomendada a instalação de pias para contemplar as necessidades de acordo com o recomendado pela OMS, orçamento do material de consumo necessário como papel toalha, sabonete e álcool gel , instalação de cartazes informativos e de orientação.

\section{Sensibilização da Equipe:}

Foram realizadas reuniões para sensibilização da equipe em relação ao tema. Foram realizadas discussões em grupos gerando espaço para que os profissionais refletissem sobre os problemas enfrentados no cotidiano e tecer criticas e comentários em relação às dificuldades para a implantação do projeto.

\section{Apresentação e envio do protocolo para os coordenadores e aula de treinamento:}

O protocolo escrito baseado no protocolo do Ministério da saúde. Aula didática para treinamento em sala de aula e impressão e plastificação do material para uso nas enfermarias em momentos de treinamento em campo de prática.

\section{Elaboração e confecção do material de divulgação do treinamento e de material de apoio:}

A divulgação do treinamento foi realizada de forma adequada através de cartazes informativos $\mathrm{m}$ locais de circulação das equipes. Os treinamentos foram marcados em datas que possibilitaram o treinamento de todos os colaboradores.

\section{Identificação da equipe de facilitadores e data do treinamento:}

Os facilitadores que foram participar do treinamento, bem como os horários de sua participação foram planejados previamente.

\section{Avaliação diagnostica do número de oportunidades de higiene de mãos da equipe:}

O número de oportunidades de Higienização das Mãos avalia as principais oportunidades de higienização das mãos para os profissionais da saúde durante o cuidado assistencial (antes do contato com o paciente, antes de realizar procedimento 
limpo e asséptico, após riscos de contato com fluidos corpóreos, após tocar o paciente e após tocar superfícies próximas ao paciente).

Foi instruído sua avaliação no período basal imediatamente antes da campanha e após a campanha. Foi seguido o manual de observadores para instruir a equipe da maneira correta de observar o número de oportunidades da ANVISA (Figura 1).

Figura 1 - Modelo do folheto que foi utilizado para anotar as oportunidades de higiene de mãos

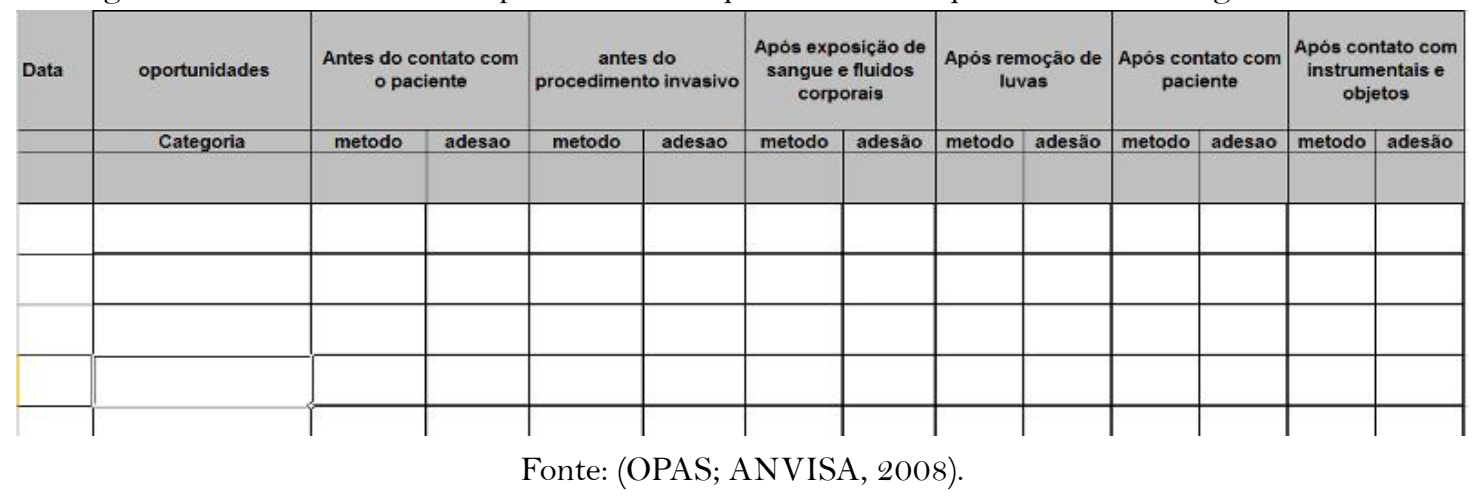

\section{Elaboração do resultado do treinamento:}

Após a conclusão do treinamento, o coordenador do NSP consolidava os dados do treinamento identificando os colaboradores que não participaram do treinamento para serem chamados para novo evento.

\section{Elaboração da ficha do indicador, análise dos resultados e plano de melhoria:}

Foram construídos os indicadores para o acompanhamento da adesão do protocolo. Os seguintes indicadores foram recomendados:

- Indicadores Obrigatórios:

a) Consumo de preparação alcoólica para as mãos: monitoramento do volume de preparação alcoólica para as mãos utilizadas para cada 1.000 pacientes-dia.

b) Consumo de sabonete para as mãos: monitoramento do volume de preparação alcoólica para as mãos utilizadas para cada 1.000 pacientes-dia.

- Indicador recomendável:

a) Percentual de adesão: número de ações de higiene das mãos realizados pelos profissionais de saúde / número de oportunidades ocorridas para higiene das mãos, multiplicado por 100.

A ficha do indicador contém as seguintes informações:

- $\quad$ Nome do Indicador

- Objetivo

- Fórmula do Cálculo do Indicador

- Análise e Interpretação do Indicador 
- Método

- Meta

- Análise do resultado e plano de melhoria

Após resultado do indicador, uma análise e proposta de melhoria foi realizada. Para isso utilizou-se a ferramenta PDCA. Esta ferramenta de qualidade auxilia na melhoria dos processos. É composto das seguintes etapas:

- P: do verbo "Plan", ou planejar.

- D: do verbo "Do", fazer ou executar.

- C: do verbo "Check", checar, analisar ou verificar.

- A: do verbo "Action”, agir de forma a corrigir eventuais erros ou falhas.

Desta maneira a cada resultado do indicador um novo ciclo do PDCA deve ser executado para a melhoria do processo.

\section{CONSIDERAÇÕES FINAIS}

A implantação de protocolos normatizada pelo Programa Nacional de Segurança propicia a qualificação do cuidado promovendo melhora da segurança dos pacientes. O conhecimento de ferramentas de gestão e instrumentos que favoreçam a sistematização das ações necessárias leva a agilidade no processo. Publicações para a implantação dos protocolos estão disponíveis na ANVISA (ANVISA, 2008), porém todas as ações necessárias estão fragmentadas em várias publicações e ferramentas de gestão com suas finalidades não são descritas no material.

Qualidade implica em melhoria dos processos, sendo resultado do esforço compartilhado de toda a equipe, isto requer mudança de postura comportamental do grupo. Por isso, a sensibilização e o envolvimento de toda a equipe são fundamentais para a mudança de paradigma. O conhecimento sobre ferramentas de gestão de risco, protocolos de segurança e demais instrumentos que favorecem a incorporação de novas rotinas e promovam a cultura da segurança do paciente, são de grande valia para o aprimoramento dos serviços de saúde.

Através da sistematização das ações apresentadas, contribuiu-se para a implantação adequada de uma das metas primordiais para a segurança do paciente. A partir do diagnóstico realizado do nível de higiene de mãos, as ações foram implementadas para a sua melhoria e seguimento do protocolo estão realizadas para o seu monitoramento

Desta forma, esta publicação poderá contribuir com embasamento teórico para que as equipes de NSP consigam transformar palavras em ações, como dito por Florence Nightingale. 


\section{REFERÊNCIAS}

ANVISA. Agência Nacional de Vigilância Sanitária. Estratégia Multimodal de melhoria da higienização das mãos. Disponível em: www.anvisa.gov. Acesso em: 08 dez 2016.

ANVISA. Manual para observadores: estratégia multimodal da OMS para a melhoria da higienização das mãos. Organização Mundial da Saúde. Brasília: Organização Pan-Americana da Saúde, 2008. Disponível em: http://www.anvisa.gov. br/servicosaude/controle/higienizacao_oms/manual_para_observadores-miolo.pdf. Acesso em: 28 jul 2018.

ANVISA. Agência Nacional de Vigilância Sanitária. Segurança do Paciente em Serviços de Saúde: Higienização das Mãos. Agência Nacional de Vigilância Sanitária. Brasília: Anvisa, 2009.

ANVISA. Agência Nacional de Vigilância Sanitária. Manual de segurança do paciente: Higienização das Mãos. Brasília, 2008.

ANVISA. Segurança do Paciente: Relatório sobre Autoavaliação para Higiene das Mãos. Disponível em https://www20.anvisa.gov.br/segurancadopaciente/index. $\mathrm{php} / \mathrm{publicacoes/category/higienizacao-das-maos}$

BARALDIM.M., PADOVEZE M.C. Higienização das Mãos: a evolução e o atual “Estado da Arte”. J InfectControl. V. 4, n.3, 2015.

BATHKE J, CUNICO PA, MAZIERO ECS, CAUDURO FLF, SARQUIS LMM, CRUZ EDA. Infraestrutura e adesão à higienização das mãos: desafios à segurança do paciente. Rev Gaúcha Enferm. V.34. n.2, p.78-85, 2013.

BELELA-ANACLETO A.S.C., PETERLINI M.A.S., PEDREIRA M.L.G. Hand hygiene as a caring practice:a reflection on professional responsibility. Rev. Bras. Enferm. V.70, n.2, p.442-5, 2017.

BRASIL. Ministério da Saúde. Gabinete do Ministro. Portaria do MS $\mathbf{n}^{\circ} 529$ de 25 de julho de 2013. Estabelece as normas para o programa Nacional de Segurança do Paciente. Disponível em: http://bvsms.saude.gov.br/bvs/saudelegis/gm/2013/ prt0529_01_04_2013.html.

BRASIL. Ministério da Saúde. Gabinete do Ministro. Portaria do MS n ${ }^{\circ} \mathbf{1 . 3 7 5}$ de 09 julho de 2013. Aprovam os protocolos básicos de segurança do paciente. Disponível em: http://bvsms.saude.gov.br/bvs/saudelegis/gm/2012/prt1375_03_07_2012. html 
BRASIL. Ministério da Saúde Portaria n. 2616, de 12 de maio 1998. Dispõe sobre a regulamentação das ações de controle de infecção hospitalar no país. Diário Oficial daUnião. Brasília, 13 maio 1998.

CARPINETTIL C.R.Gestão de Qualidade:conceitos e técnicos. 2 edição: São Paulo, 2012.

FIGUEIREDO M.L, D’Innocenzo M. Dificuldades encontradas pelos gestores de instituição de saúde na utilização da metodologia gestão por processos. RAS, v. 13 n. 50, Jan-Mar, 2011.

HAAS, J.P.; LARSON, E.L. Measurement of compliance with hand hygiene. Journal of Hospital Infection, v.66, n.1, p.6-14, 2007.

HADDAD R.E., GIORDANI A.T, EZAIAS G.M. et al. Técnica De Higiene Das Mãos E Eficiência De Degermantes Na Prevenção De Infecções Hospitalares. Rev enferm UFPE on line. V. 10 n. 2 p.: 562-7, 2016.

MARRA A.R., EDMOND M.B. New technologies to monitor healthcare worker hand hygiene.ClinMicrobiol Infect. V.20, p.29-33, 2014.

MARRA, A.R. Avanços no controle das infecções. Einstein. V.14, n.1, p.108-9, 2016.

NEVES Z.C.P.D. E COLS. Relato de experiência: utilização de cartazes como medida de incentivo à higienização das mãos. Rev. Eletr. Enf. V.11, n., p. 738-45, 2009.

OLIVEIRA, A.C.; PAULA, A.O. Monitoração da adesão à higienização das mãos: uma revisão de literatura. Acta paul. Enferm.v. 24, n. 3, p. 407-413, 2011.

PINTO, F.O.P., BAPTISTA, M.A. Higienização das mãos: hábitos, obstáculos e a técnica desenvolvida pelos discentes do $6^{\circ}$ ano de medicina e do $4^{\circ}$ ano de enfermagem de um hospital escola. ArqCienc Saúde. V.17, n.3, p.117-21, 2010.

PRIMO M.G.B., RIBEIRO, L.C.M., FIGUEIREDO L.F.S., SIRICO S.C.A., SOUZA M.A. Adesão à prática de higienização das mãos por profissionais de saúde de um Hospital Universitário. Rev. Eletr. Enf. V. 12 n 2. p.266-71, 2010.

PITTET, D.; ALlEGRANZI, B.; SAX, H.; DHARAN, S.; PESSOA-SILVA, C.L.; DONALDSON, L.; BOYCE, J.M; WHO Global Patient Safety Challenge, World Alliance for Patient Safety. Evidence-based model for hand transmission during patient care and the role of improved practices. Lancet Infectious Diseases, v. 6, n.10, p. 641-52, 2006. 
RIBEIRO F.D.O. et al. Logical strategy for improving health hygienic practices among health professionals. Journal of Nursing UFPE on line. V. 11, n. 10, p. 39713979, 2017.

SOUSA, E. C. P., SILVA, F. L. Conhecimento E Adesão Da Prática De Higienização Das Mãos Dos Profissionais Da Saúde: Revisão Bibliográfica. Rev. Saúde em Foco. Teresina, v. 3, n. 1. p. 84-93,2016.

WORLD HEALTH ORGANIZATION. Hand Hygiene Self-Assessment Framework: Introduction and user instructions, 2010. Disponível em: http:// www.who.int/gpsc/5may/tools/HHSA_framework_2011-PORTUGUESE.pdf. Acesso: em 08 dez 2016. 